\title{
Thermographic diagnostics for detecting malfunctions in TV
}

\author{
by A. Stoynova, B. Bonev
}

Technical University of Sofia, 8, Kl. Ohridski str., Sofia, Bulgaria, ava@ecad.tu-sofia.bg

\begin{abstract}
Fast and accurate diagnosing of TVs malfunctions is important in the repair activities. Two different approaches of failure detection are examined and compared based on different criteria for efficiency - the classic model with built-in self-diagnosis software and the thermographic diagnostics with infrared camera. It is shown how thermal infrared diagnostics can detect defect components, impossible to detect with built-in diagnostics and memory error recording software.

Experimental results of thermographic diagnostic of LCD TVs are presented. The saving of time and money for sophisticated diagnostic tests and significant repairs by using thermographic approach is confirmed.
\end{abstract}

\section{Introduction}

Infrared thermography has become a powerful tool for designing, manufacturing and repairing PCBs. As the engineers are pressed to design as small as possible PCBs, the density of the elements increases and this leads to problems with the heat they emit.

Infrared thermography is increasingly successful as an effective means of diagnosing malfunctions in electronic circuits [1 - 6]. Diagnosis and fault detection is the most important and responsible part of the repair of modern LCD TVs [7]. Fast and accurate diagnosis is the basis for quality repair.

A wide range of TVs' problems and failures are accompanied by a temperature change that can be detected by thermal measurements. This makes it possible to minimize the risk of damage to a specific electronic unit or element and also to provide reliable tool for repairs. As a further advantage, it is possible to specify the exact location of the problem area (hot spot) of the PCBs and reduce the time to detect the failure [8-10].

In the paper, an approach for diagnosing some types of hardware faults in modern TVs with 2D thermal measurements by infrared camera is presented. The purpose is to examine diagnostic procedures on electronics items that are most likely heat-laded. The effectiveness of performing a thermographic diagnosis is based on the reliable problem element detection and the time it takes to perform the procedure compared to time of diagnostic applying service regimes. To prevent different unwanted signals (e.g., from dead pixels, from surrounding environment of the measurement setting), the thermal image sequences are pre-processed and the subsequent processing is performed.

Two different approaches for LCD TVs' diagnosing and detecting malfunctions are presented. The first is the classic model with built-in self-diagnosis software and memory error logging. In this case, the diagnosis is carried out with multiple measurements of voltages at control points. The second method - thermographic diagnostics with the use of an infrared camera is examined in detail. The advantages of the thermographic analysis used to detect some hardware malfunctions in the LCD TV are noted.

\section{Possibilities for diagnosis of the LCD TV electronic modules}

Trend of TVs are going slim and compact style design to meet the demands of the market. The normal performance TV image quality is influenced largely by thermal regime. As power consumption increases, electronics elements can heat up during normal operation. The surface or case temperature of elements is related to the power dissipation in the components.

Ones of the most popular models of TVs in Bulgaria with same block diagrams, are chosen to be studied: Philips 40PFL3107H and Philips 42PFL6008K. Research has been conducted in an authorized service centre for this TV brand.

40PFL3107H/12 is a Full HD LED TV with a screen resolution of 1920 x 1080 pixels, screen diagonal 102cm, brightness $400 \mathrm{~cd} / \mathrm{m}^{2}, 100,000: 1$ dynamic contrast ratio, $100 \mathrm{~Hz}$ scanning frequency [7].

42PFL6008K/12 e Full HD LED 3D Smart TV with screen resolution of $1920 \times 1080$ pixels, screen diagonal $107 \mathrm{~cm}, 400 \mathrm{~cd} / \mathrm{m}^{2}$ brightness, 500,000: 1 dynamic contrast, $500 \mathrm{~Hz}$ picture scanning frequency, 2D - 3D conversion, adjustable 3D depth, free internet browser, online applications: Skype, You Tube and more. LCD Panel.

Fig. 1 shows block diagram of such types LCD TV. It includes three main blocks: power supply; main board and 


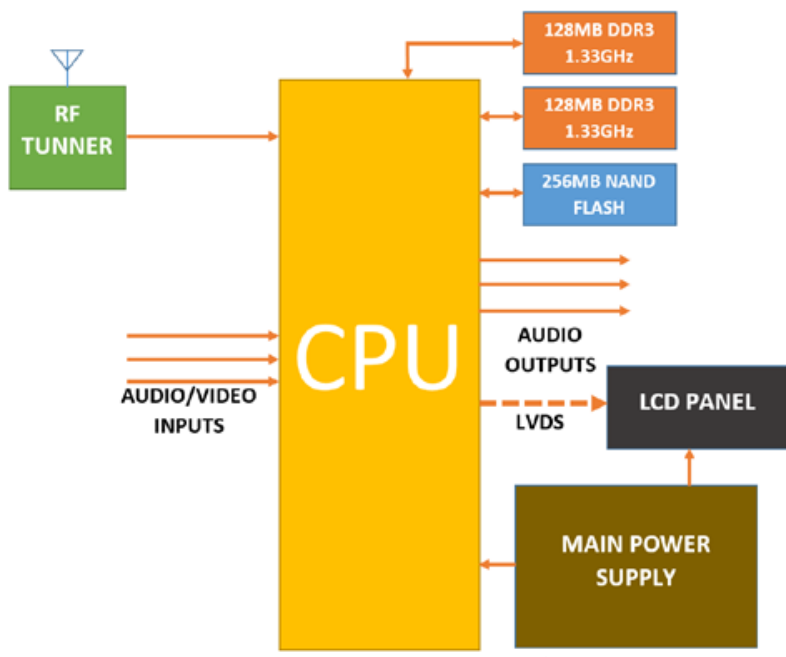

Fig. 1. Block diagram of LCD TV

The power supply unit consist of several basic modules: AC-Input mains filter, Power Factor Corrector (PFC), Low power standby supply, Platform power supply, and Display power (including LED backlight) supply.

Damages related to a power supply unit are mostly due to power supply problems. Poor quality or fluctuating power supply can often cause power surges or sages, spikes and voltage fluctuations. The malfunctions of Platform and Display power supply are easy to detect using a voltmeter and an ohmmeter. They are built of relatively inexpensive components - diodes, transistors, resistors and capacitors and because of that it is not subject of research in this paper. complex.

The main board of the TV is a multi-layer PCB. It contains chips in BGA package and therefore the diagnosis is

In main Board are located RF tuner DVB-T/C MPEG4 and all inputs:

- Cl card slot;

- video signal inputs (HDMI, SCART, PC VGA, YPbPr, CVBS);

- audio signal inputs;

- USB input for external storage device;

- Ethernet input.

Signals from all inputs are processed and decoded in the CPU MT5366, which controls the LCD display via built in LVDS Transmitter and the audio amplifier STA381BW. The CPU processes also signal coming from remote control and from TV keyboard. The main board of the TV contains also following memories: two RAM DDR3 128MB and NAND Flash 256MB.

Damages in TV main board are mainly related with CPU and the peripheral interface. There are software and hardware types of failures. Malfunctions can be appear due to over voltages from atmospheric sources, degraded cooling conditions, etc. In main board, the correct diagnosis is extremely important and infrared thermography as diagnosis tool for the detection of malfunctions can be used.

The TV's LCD display cannot repair in the service centre conditions. When failure in LCD is appeared, the LCD display or the entire TV is replaced. The display defects in it are mainly due to improper use related to bending and transport problems [8]. But the temperature distribution on the LCD display can be evaluated at steady state using infrared camera.

The standard diagnosis approach begins with booting the TV in service mode (if possible). There are mainly three types of service modes:

- Service Default Mode (SDM);

- Service Alignment Mode (SAM);

- Customer Service Mode (CSM).

The TV can be booted up in SDM mode in two ways: with remote control test of button combination or with connecting a SDM control point to GND, shown on Fig.2.

The TV can also be booted up in SAM and CSM modes with a combination of buttons on the remote control. When the SAM mode is enabled, the diagnostic of the Main Board starts with reading the errors in the Error Buffer. There is an LED - flashing procedure for level board repair LAYER 1 errors. LAYER 2 errors require component level repair in service centre conditions. (see Table 1). LAYER 1 errors are labelled with one-digit numbers, and LAYER 2 errors are labelled by two-digit numbers. Some of the error codes are shown in Table 1:

- Error 13 (I2C bus M3, SSB + SRF bus blocked)

- Error 14 (I2C bus M2, BE bus blocked)

- Error 18(I2C bus M1, FE bus blocked)

The best way to further diagnosis is by measuring in control points, when these errors occurs.

- Error 15(Fusion doesn't boot) 
For example, Error 15 indicates that the main CPU can't read its instructions. This error is due to hardware problem with the CPU and the CPU peripheral:

- problems with power supply;

- CPU is fully damaged;

- there are no connection between Mips and standby processor;

- problems with RAM memory, etc. instructions

Other major reasons for this error can be hardware issues pointing to DDR's and reading the CPU's start-up

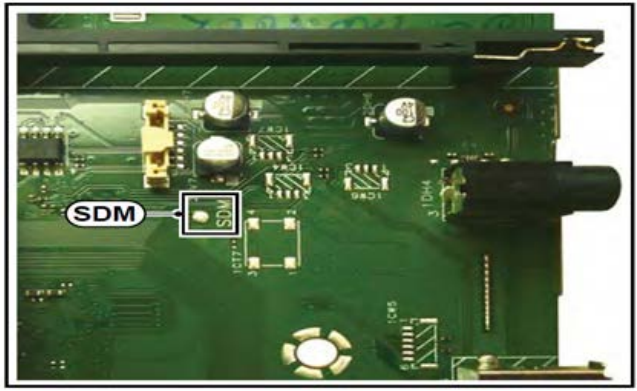

Fig. 2. Control point SDM

Table 1. A sample of error codes, which can be read during SAM

\begin{tabular}{|c|c|c|c|c|c|c|c|}
\hline Description & Layer1 & Layer2 & Monitored by & $\begin{array}{l}\text { Error/ } \\
\text { Prot }\end{array}$ & $\begin{array}{l}\text { Error Buffer/ } \\
\text { Blinking LED } \\
\end{array}$ & Device & $\begin{array}{l}\text { Defective } \\
\text { Board }\end{array}$ \\
\hline $\mathrm{I}^{2} \mathrm{CM} 3$ & 2 & 13 & MIPS & $E$ & $\mathrm{BL}$ & SSB & SSB \\
\hline $\mathrm{I}^{2} \mathrm{CM} 2$ (BE bus) & 2 & 14 & MIPS & $E$ & $\mathrm{BL}$ & SSB & SSB \\
\hline $\begin{array}{lll}\text { PNX (Fusion) doesn't } \\
\text { boot }\end{array}$ & 2 & 15 & Stby $\mu \mathrm{P}$ & $P$ & $\mathrm{BL}$ & Fusion & SSB \\
\hline $12 \mathrm{~V}$ & 3 & 16 & Stby $\mu \mathrm{P}$ & $\mathrm{P}$ & $\mathrm{BL}$ & 1 & Supply \\
\hline $\mathrm{I}^{2} \mathrm{CM} 1$ (BE bus) & 2 & 18 & MIPS & $\mathrm{E}$ & $\mathrm{BL}$ & SSB & SSB \\
\hline$\ldots$ & $\ldots$ & $\ldots$ & & $\cdots$ & & $\ldots$ & \\
\hline NT72314 not available & 9 & 63 & MIPS & $E$ & EB & NT72314/ & QFHD \\
\hline
\end{tabular}

From the illustrated example, it can be noticed that a standard approach is preferable when have to look for software faults. However, the hardware failures diagnosis is associated with a loss of much time

Table 2 shows statistical data for most common defects in LCD TVs PHILIPS. It can be seen that most often failures occurs in TV main board. It can be easily detected the defective block (PSU, Main Board, and LCD) with using described classical diagnosis methods. It is considerably more difficult to do a component level diagnosis and to determine which element is defective especially when the problem is in the TV main board.

The package type of the CPU chip is ball grid array (BGA) and therefore classical diagnostic of it is considerably more difficult. Defects related to CPU and peripheral integrated circuits can be easily detected by thermography measurements.

Table 2. Statistical data for most common failures in different blocks of LCD TVs

\begin{tabular}{|l|l|l|l|}
\hline Block & PSU & Main Board & LCD \\
\hline Failures, \% & 25 & 60 & 15 \\
\hline
\end{tabular}

\section{Thermographic diagnostics of LCD TV}

Infrared thermography use has been steadily growing in more and more areas. The reason for this is that it allows a new level of diagnosis and problem solving. As more important advantages can be mentioned:

- Avoids removing and assembling parts of the PCB that do not need to be repaired. It is only repaired, what is needed to be repaired;

- Allows quick troubleshooting before the failure occurs.

When the nominal temperature values of the PCB elements are known, the infrared camera can image the variations to the specification. These variations may be towards lower or higher temperatures. Based on these monitored data, quick decisions can be taken for immediate or postponed repairs of specific elements.

Emissivity of many PCBs' components differ widely, some of them have a very low emissivity $(\varepsilon<0.3)$. As the emissivity is lower so the measurement error is higher (a camera with a range of $7.5 \mu \mathrm{m} \div 13 \mu \mathrm{m}$ is used). To compensate the difference in PCB component emissivity, the following approaches have been used:

- software compensation of individual emissivity of the components; 
- comparison with reference PCB.

Infrared camera FLIR TermaCAM SC640 is used for thermal measurement $\left(45^{\circ}\right.$ lens). The IR camera is connected via IEEE1394 interface. Infrared image processing are performed with software ResearchIR and selfdeveloped software for thermal histograms analysis.

Photo of the experimental setup is shown on Fig. 3.

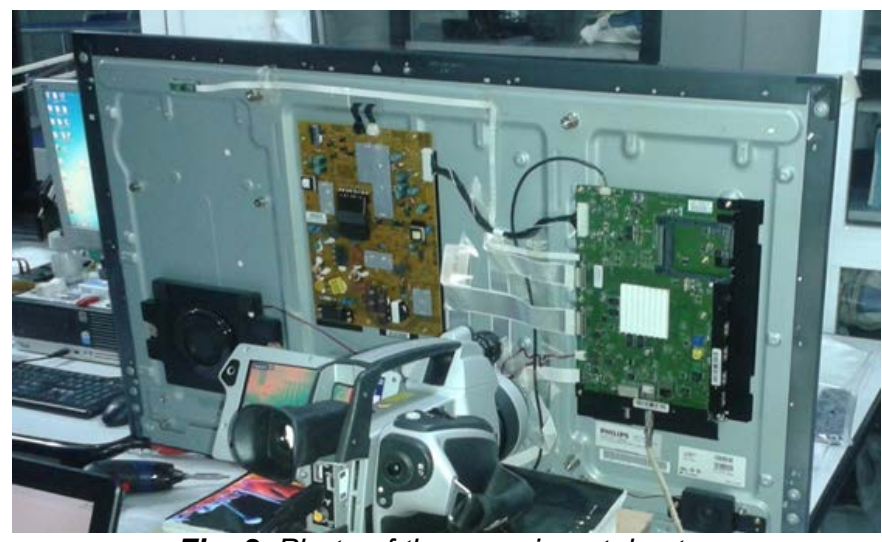

Fig. 3. Photo of the experimental setup

Fig. 3 shows thermography study at the diagnosis of the defective TVs. The infrared camera captures TV main board and more specifically CPU area. The ambient temperature, relative humidity and the distance between IR camera's lens and tested PCB are measured in order to compensate their impact on temperature measurement accuracy.

\section{Experimental results and analysis}

42PFL6008K / 12 TVs in standby mode were tested, with an expected power consumption of $0.3 \mathrm{~W}$. Fig. 4 shows thermograms 48 seconds after turning on standby mode. The change of temperature is monitored on the following marked items: SP01 - DC / DC converter 7UU1; SP07 - DC / DC converter 7UU2; AR01 - CPU 7J00. The SP01 - SP08 shows the maximum temperatures and AR01 * is set to display the average temperature. LI01 * is set to indicate the maximum temperature. Since AR01 and LI01 are on the heat sink's surface, the sign "*" indicates that the emissivity correction has been made in the thermogram.
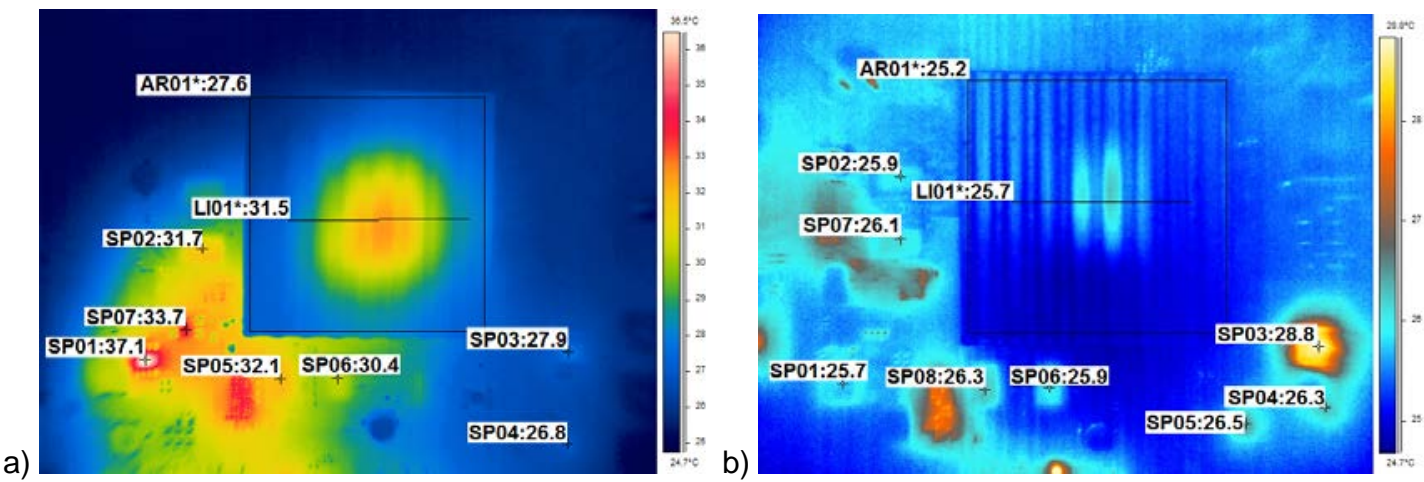

Fig. 4. Thermograms $48 \mathrm{~s}$ after turning on standby mode for: a) TVs with malfunctions TVs; b) reference TVs

The temperature-time diagrams of the marked points, area and line are shown in Fig. 5. The cursor displays the time of standby mode turning off. The diagram shows a non-specific heating at point SP01 (red colour) - DC/DC convertor 7UU1. His chart reaches the highest value compared to the other areas studied. 48 seconds after turning on the TVs, the temperature at this point reaches $38{ }^{\circ} \mathrm{C}$, while the CPU temperature at this point is $310 \mathrm{C}$. It can be assumed that the problem of the TV is due to a DC / DC converter 7UU1 defect or related components. From the comparative thermographic analysis made between the defective and the reference TVs, we have undoubtedly come to the conclusion that the problem is due to a defect in the DC / DC converter 7UU1.

Fig. 5 shows temperature distribution over time for different marked elements. The legend is given only for commented in the paper components. On Fig. 5a is shown temperature distribution for $15 \mathrm{~s}$ after turning on standby mode. We monitor temperature change on the following marked elements: SP01 - DC / DC converter 7UU1; AR01 - CPU 7J00. The temperature time diagrams of the marked areas are shown in Fig. 5b and Fig.5b. At 13:12:41 h the TVs is switched on in standby mode, and 12 seconds later at 13:12:53 h, a "power on" signal is output from the remote control 
and the TVs is switched to normal operating mode. We are interested in the first 12 seconds when the TV is in standby mode and the temperature value at SP01 DC / DC converter 7UU1. It is seen that the temperature is equal to $26.7^{\circ} \mathrm{C}$ and does not change over time.

For the ROI of the thermographic measurement, we select the following components from the Main Board of the TVs 40 PEL 3107 with Al heat sink:

- Point SP01 - DDR U4003 - maximum temperature ${ }^{\circ} \mathrm{C}$;

- Point SP02 - DDR U4002 - maximum temperature ${ }^{0} \mathrm{C}$;

- Point SP03 - Stabilizer U7006 - maximum temperature ${ }^{0} \mathrm{C}$;

- Point SP04 - DC / DC U7004 - maximum temperature ${ }^{0} \mathrm{C}$;

- Point SP05 - Induction L 7101 - maximum temperature ${ }^{\circ} \mathrm{C}$;

- Maximum temperature distribution on line LI02 - processor heat sink MT5366.

On Fig.6a, b (LI01 and LI02) the thermogram and the temperature values in steady state mode are shown, respectively. On Fig.6c, d temperature distribution over time is shown during the transient period after turning on standby. Four minutes after turning on a steady state mode an equilibrium have been reached. The average temperature of the heat sink is stabilized to $41.2^{\circ} \mathrm{C}$. The temperature distribution is evenly across the heat sink area and no problem areas are detected. For this case it can be concluded that the processor is working normally (Fig.6c - see LI01 and LI02). After turning on HD (High-Definition) mode the surface temperature on the heat sink starts to rise and after 5 min it reach stabilization to about $56^{\circ} \mathrm{C}$ (Fig. $6 \mathrm{~d}$ - see LI01 and LI02). On the Fig.6 legend is shown only for commented elements of the Main Board.

One another approach for faster temperature mode evaluation of the PCB elements is used.

Thermal histograms were used to compare two thermograms from different sources or with different geometric parameters. The thermal histograms also provide useful information in the continuous monitoring and analysis of the temperature changes of a selected object. For this purpose, an algorithm has been developed with the means of the AGE environment, which analyses digital thermograms and derives the temperature information at the pixel level. An important feature of the proposed algorithm for composing a thermal histogram in AGE is that it works with colour samples that are automatically selected evenly from the colour palette. The density of the colours in the histogram is grouped and approximated to the nearest colour sample. For better performance, each sample is coloured with the pixel colour of the infrared image palette. The parallel program also uses one additional node for automatic parallel imaging of image sequences. After running the program using the AGE simulator, all files in the specified directory are processed in parallel.

For example, when the problem is in the DC / DC converter 7UU1, the temperature histograms from Fig.7a and Fig.7b are compared. Fig.7c shows the temperature histograms for the converter for the sequence of 5 thermograms after switching on the TVs. The appearance of the peak after the third minute is indicative for the presence of malfunction in the DC/DC converter.

On Fig. 8 the thermal histogram is shown for the case of processor without failure. It can be seen the selected field for analysis on its heat sink. The program for thermal histogram analysis is shown. It can be seen the graphical window with main menu.

Although the TFT LCD of TVs are extremely rarely diagnosed for failure in the service centre, the proposed software can also be used to evaluate the temperature field in different areas of the screen [11]. Such a diagnostic in this case is more of a preventive nature.

Fig. 9 shows the 3D temperature distribution (front display \& cell surface) and the thermal histogram of the LCD. The highest measured temperature (about $45^{\circ} \mathrm{C}$ ) at steady state mode on the TFT LCD is on the Bracket surface.

The requirements for camera calibration, environmental parameters measurements and corrections, as well as the individual steps in performing a correct measuring procedure are not commented in this paper.

We can also use image recognition algorithms during the infrared diagnostics, but given the nature of the work, the efficiency of such an approach is not high in service centres (a wide variety of TVs, modular-level and not component-level repairs, and more frequent occurrence of software than hardware malfunctions) [12]. 


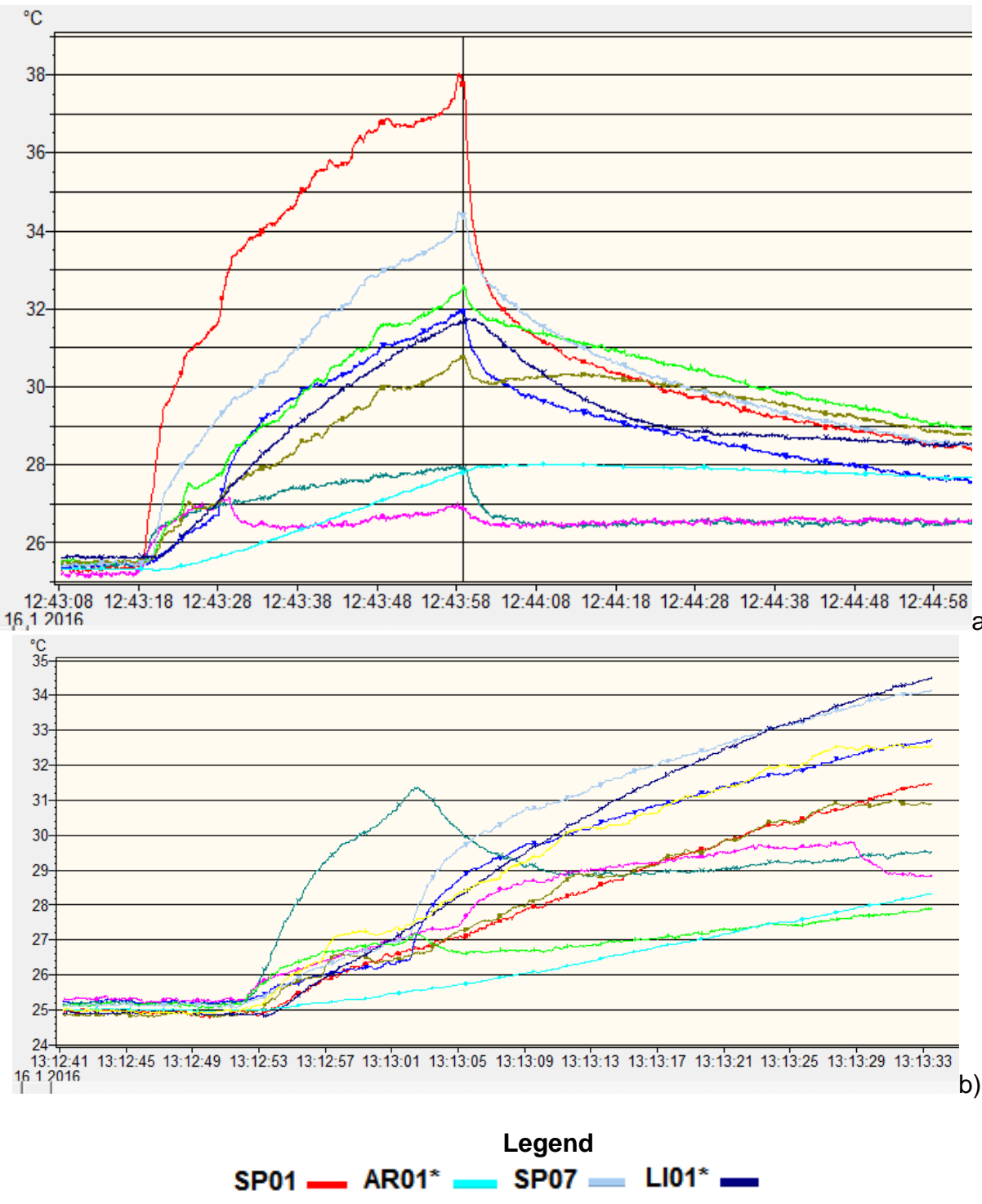

Fig. 5. Temperature distribution for marked areas in Fig. 4: a) defective TVs and b) reference TVs

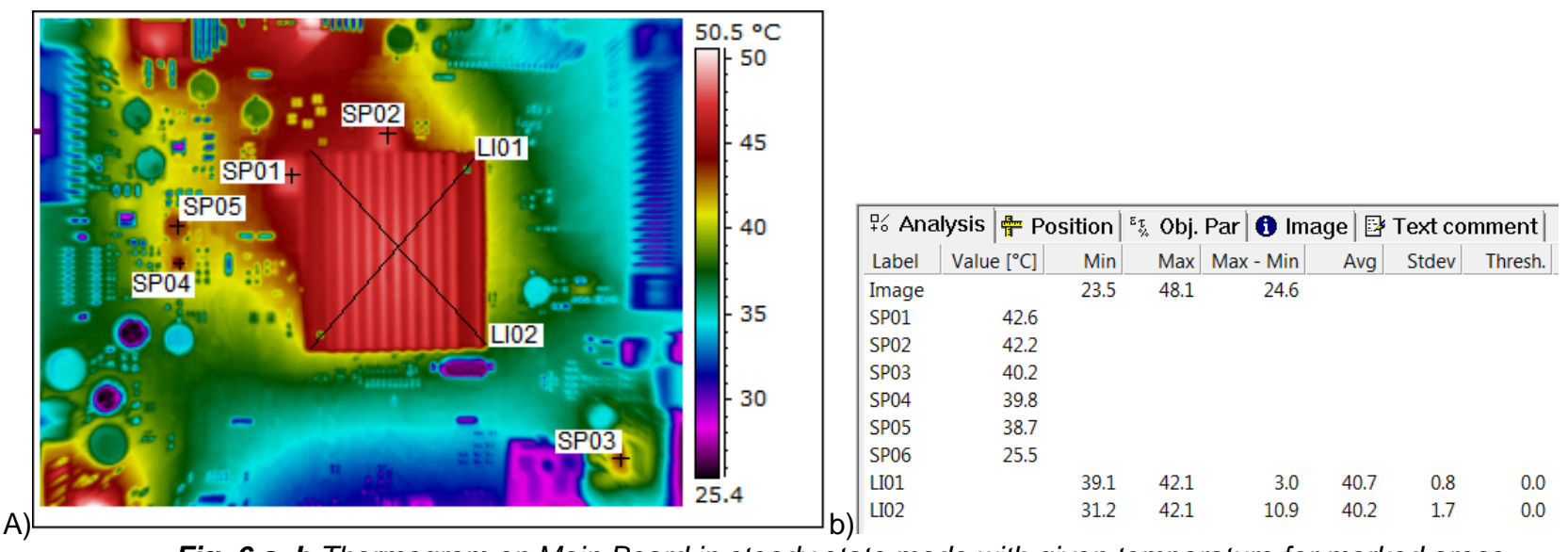

Fig. 6 a, $\boldsymbol{b}$ Thermogram on Main Board in steady state mode with given temperature for marked areas 


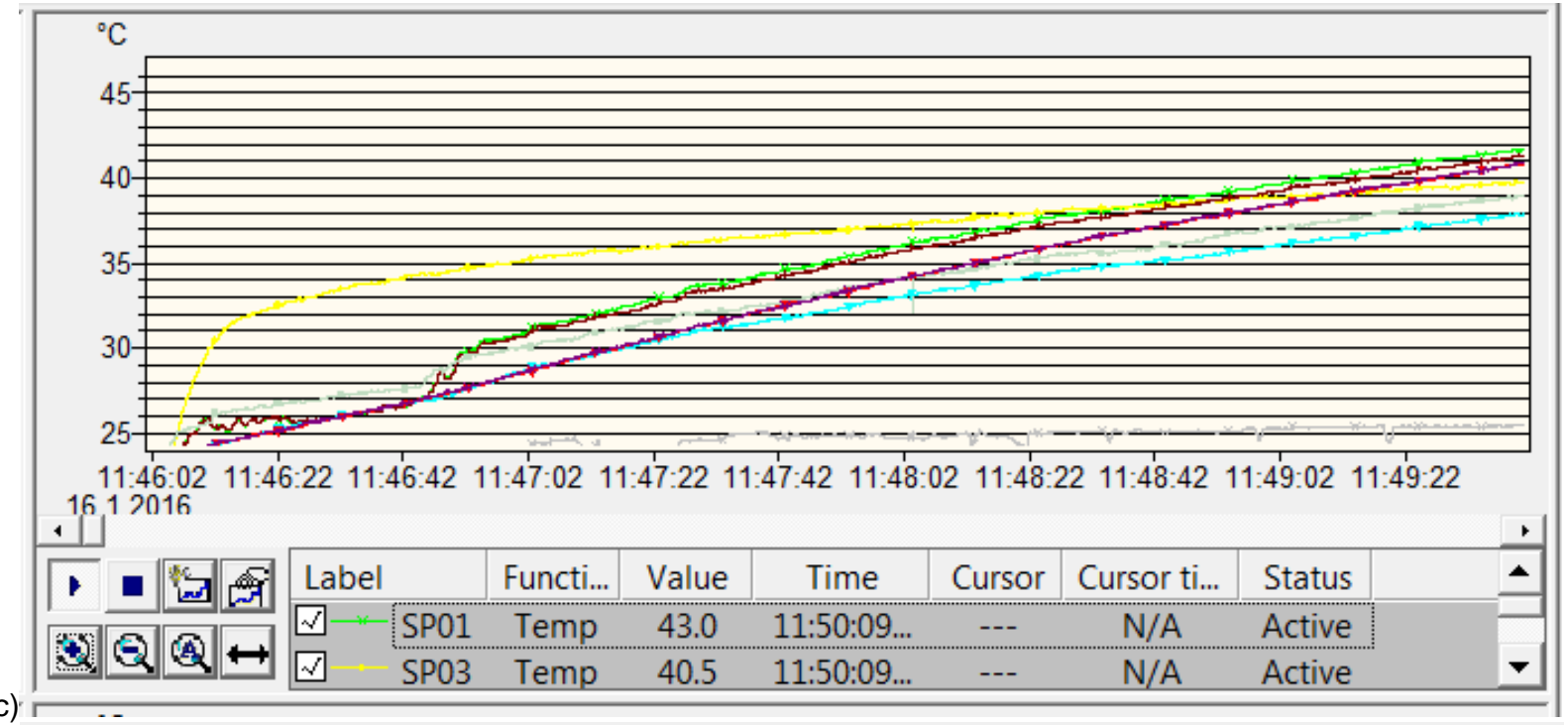

c)

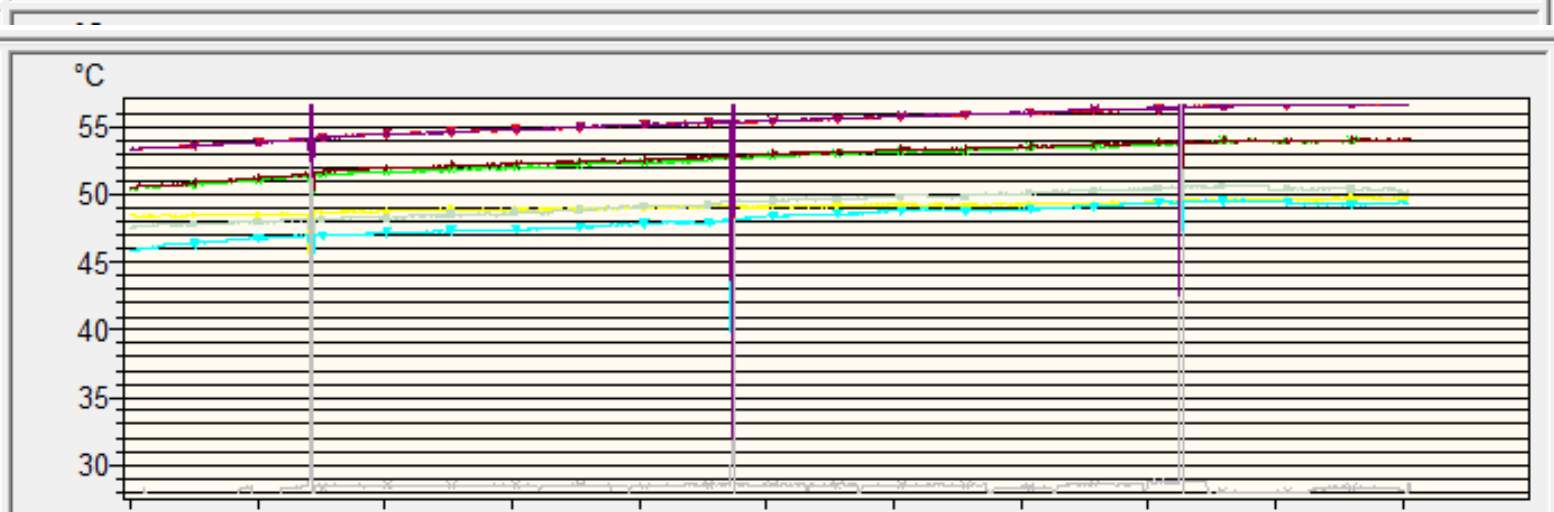

12:03:15 12:03:55 12:04:35 12:05:15 12:05:55 12:06:35 12:07:15 12:07:55 12:08:35 12:09:15 12:09:55 1612016

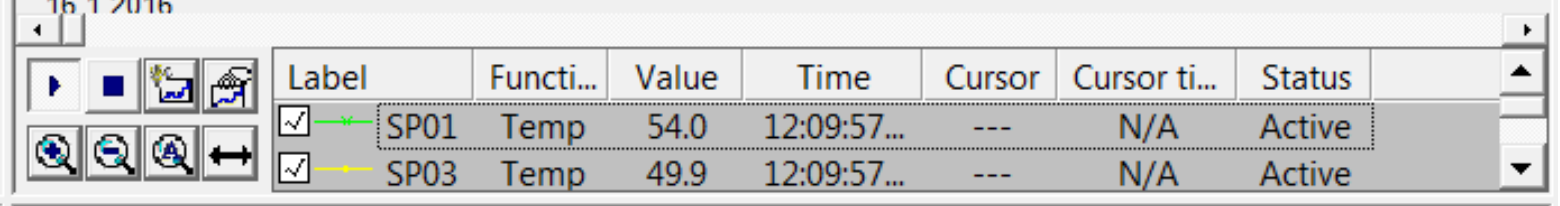

d)

\section{Legend}

$\mathrm{SP} 01-\mathrm{SP} 02-\mathrm{SP03}-\mathrm{SP04}$ SP05 $\mathrm{LI01}$ - LI02

Fig. 6 c, d. Temperature distributions - c) stby power; d) HD power
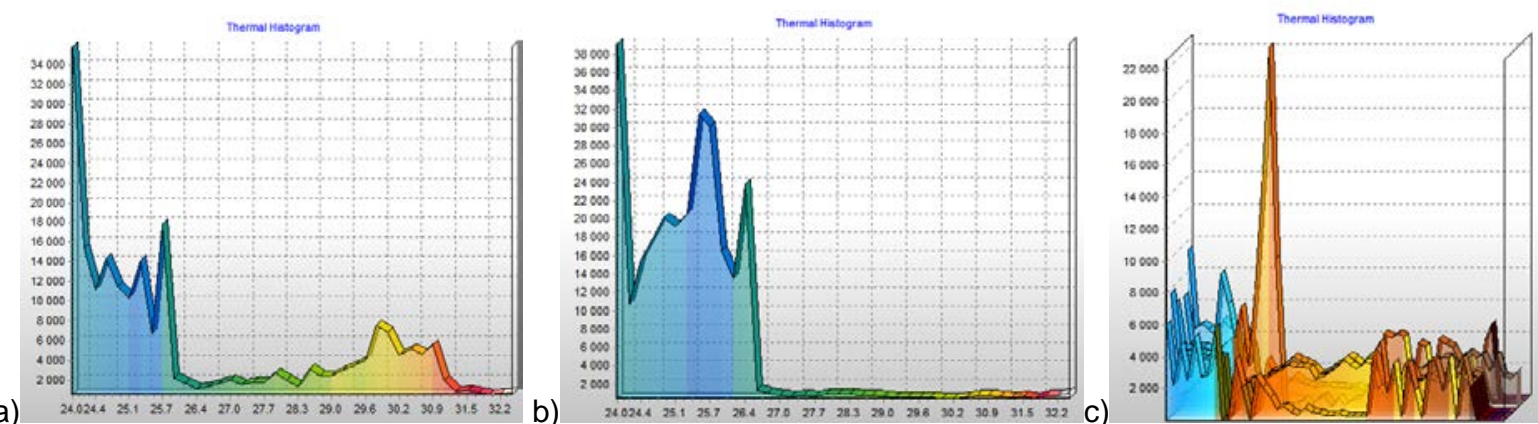

Fig. 7. Thermal histograms' of DC / DC converter 7UU1: a) with failure; b) without failure; c) with malfunction 


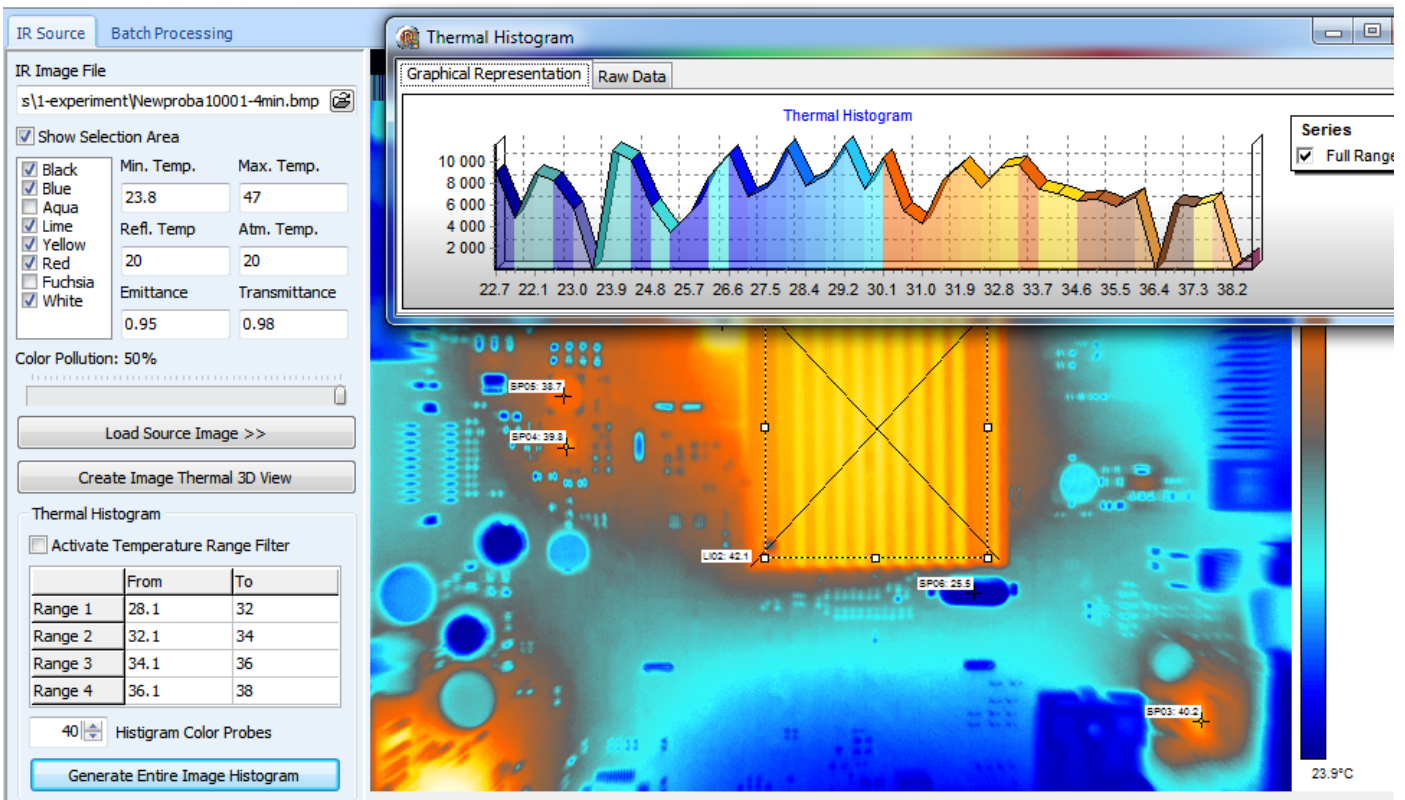

Fig.8. View of the program for thermal hystogram creation and analysis with the thermal hystogram of reference processor
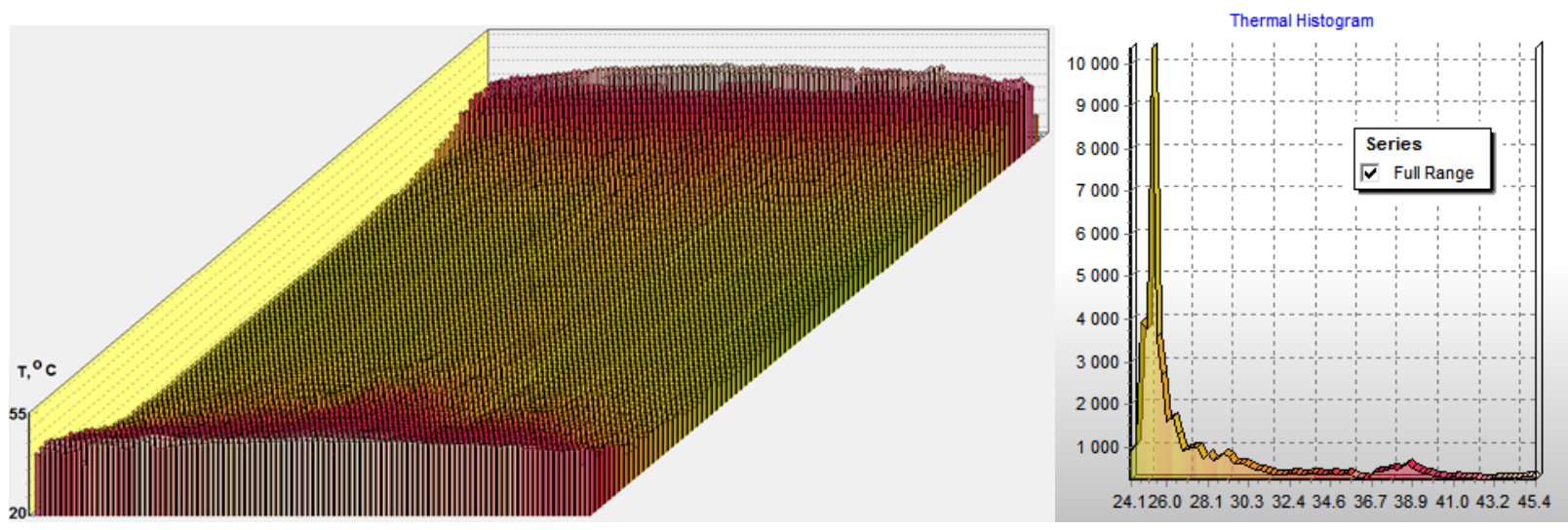

Fig.9. 3D thermal view and thermal histogram of TFT LCD at steady state

\section{Conclusions}

A wide range of problems and failures in LCD TVs are accompanied by a temperature change that can be detected and measured by infrared camera. This makes it possible to minimize the risk of failure to a specific electronic unit and also to provide reliable tools for repairs.

An approach is proposed for comparison of thermal histograms, which is also useful in the continuous monitoring and analysis of the temperature changes of the main boards of TVs.

It is possible to measure the temperature of each element of the board and to evaluate its performance. It is also possible to diagnose a block as a separate unit. Thermographic imaging of LCD TVs helps to quickly and accurately to be detected the defective component or block.

Thermal diagnostics can detect factory defects and prevent more damage.

\section{ACKNOWLEDGMENT}

The author would like to thank for the support of National Science Fund under which Project No. DN $17 / 16$ the present work was conducted. 


\section{REFERENCES}

[1] Breitenstein O., Lock-in IR Thermography for functional testing of electronic devices, Proceedings of 7th Quantitative InfraRed Thermography conference, Belgium, pp. B.3.1-6 paper, 2004.

[2] Akole S., Kulkarni V., Thermal analysis of active electronic component with thermal Imaging. IJCA Proceedings on NCECE 2016(1), Pune, India, pp. 11-15, 2016.

[3] Petrosyants K., Kharitonov I., Popov A., Analysis of temperature-current rise in modern PCB traces by means of thermography, Proceedings of 13th International Workshop on Advanced Infrared Technology \& Applications, Pisa, Italy, pp.171-174, 2015

[4] Stoynova-A., Georgiev N., Erinin A., Bonev B., Hidden solder joints inspection used in surface-mount technology, Proceedings of 39th ISSE, Pilzen, Czech, pp. 1-6, 2016.

[5] Jadin M., Taib S., Recent progress in diagnosing the reliability of electrical equipment by using infrared thermography, Infrared Physics and Technology, Vol. 55, no. 4, pp. 236-245, 2012.

[6] Andonova A., Ivanov S., Neychev I, Kafadarova N., Research of Active Gate Drivers for MOSFET by Thermography, New aspects of automatic control, modeling and simulation ACMOS, pp. 29-31, 2010.

[7] Service manual TPM9.2ELA, http://www.atyourservice.philips.com

[8] Wagh C., Baru V., Detection of faulty region on Printed Circuit Board with IR thermography, International Journal of Scientific \& Engineering Research. - Vol 4, Issue 11, pp. 1-4, 2013.

[9] Swapnil P., Shah D., Fault diagnosis in electrical and electronic equipments using thermography and image processing-a survey, IJIRT. - Vol. 1, no 11 (2014), pp. 365-369, 2014.

[10] Varghese J., Singh T., Mohan S., PCB Thermal Image Analysis using MATLAB, IJRAET. - Vol. 2, no 3 (2014), pp. 46-52, 2014.

[11] İz M., Tavman I. Turgut A., A study on cooling efficiency improvement of thin film transistor liquid crystal display (TFT-LCD) Modules, Proceedings of 13th Electronics Packaging Technology Conference, Singapore, pp. 536$541,2011$.

[12] Stastny J, Skorpil V., Object recognition by means of early parser effective implementation, Proceedings of 40th International Conference TSP, Barcelona, Spain, pp.271-274, 2017. 